\title{
CONSUMPTION OF GREEN TEA OR ITS DERIVATIVE CATECHIN MAY IMPROVE NEURAL REGENERATION IN A RAT SPINAL CORD INJURY MODEL
}

\author{
๑ Rifat AKDAĞ', ๑ Özhan Merzuk UÇKUN², ๑ Ali DALGIÇ², ๑ Gülnur TAKE ${ }^{3}$ \\ ${ }^{1}$ University of Health Sciences Turkey, Bursa Yüksek Ihtisas Training and Research Hospital, Clinic of Neurosurgery, Bursa, Turkey \\ 2University of Health Sciences Turkey, Ankara City Training and Research Hospital, Clinic of Neurosurgery, Ankara, Turkey \\ ${ }^{3}$ Gazi University Faculty of Medicine, Deparment of Histology and Embryology, Ankara, Turkey
}

Objective:Tea contains antioxidant compounds from the polyphenol group known as catechins. The most abundant of catechins is Epigallocatechin3-gallate (EGCG). Epidemiological studies show that tea has a protective effect against cancer, neuronal damage after transient global ischemia and cardiovascular diseases. This study aimed to investigate a possible neuroprotective effect of EGCG in a rat spinal cord injury (SCI) model. Materials and Methods: The study was performed with 35 Albino-Wistar rats. Rats were divided into five groups: daily consumption group (intraperitoneal given ECGC $1.7 \mathrm{mg} / \mathrm{kg} /$ day), treatment groups (intraperitoneal given ECGC $5 \mathrm{mg} / \mathrm{kg} /$ day and $10 \mathrm{mg} / \mathrm{kg} /$ day), saline group and control group for 14 days prior to trauma. All groups, other than the control group, injured with a pressure of $35 \mathrm{~g} / \mathrm{cm}^{2}$ and $1-\mathrm{minute}$ compression. These operations were applied to the spinal cord at level T9-T10. In all groups, nerve samples were taken after 28 days and examined biochemically and histopathologically.

Results: In our study, daily consumption ECGC group, $5 \mathrm{mg} / \mathrm{kg}$ ECGC group and $10 \mathrm{mg} / \mathrm{kg}$ ECGC group statistically significant lower level of lipid peroxidation. Especially daily consumption ECGC group and $5 \mathrm{mg} / \mathrm{kg}$ ECGC group were positively decreased histological degeneration and oedema. Histological evaluation, white-grey matter sparing, glial scar formation, protoplasmic astrocytes' number, cavity size, also had better results in these groups.

Conclusion: In this study, it has been shown that catechin group antioxidant substances in tea have a protective effect in neuronal damage such as $\mathrm{SCl}$.

Keywords: Spinal cord injury, epigallocatechin-gallate, immunostaining, electron microscope, lipid peroxidation, green tea

\section{INTRODUCTION}

Traumatic spinal cord injury ( $\mathrm{SCl}$ ) is a serious medical condition caused by damage to the central nervous system. Complications of $\mathrm{SCl}$ are a frequent cause of morbidity and mortality and lead to motor paralysis, sensory and autonomic disturbances, for which appropriate treatment has not yet been developed ${ }^{(1)}$. $\mathrm{SCl}$ is most commonly caused by motor vehicle accidents or falls, and $\mathrm{SCl}$ victims are usually young ${ }^{(2)}$. Long-standing experimental and clinical academic works have demonstrated the major pathological changes that cause all SCl-induced signs and symptoms. The spinal cord contains many blood vessels, and like the blood-brain barrier, the microcirculation is tightly controlled by the blood-spinal cord barrier (BSCB), which is a distinct anatomical barrier ${ }^{(3)}$. After the traumatic $\mathrm{SCl}$, disruption of the BSCB, plasma and blood cell extravasation, central sensitisation of nociceptive spinal cord neurons, cell necrosis, release of inflammatory mediators, reactivation of glial cells and increased potassium and glutamate levels' neurotoxic excitatory amino acids in the extracellular space are the major changes observed in the damaged spinal cord ${ }^{(4)}$. SCl triggers the beginning of a response with a series of biochemical changes in the spinal cord. Increased myeloperoxidase activity, neutrophil infiltration and the release of inflammatory mediators cause elevation of lipid peroxidation level ${ }^{(5)}$. Lipid peroxidation is a toxic chain reaction that progresses with positive feedback ${ }^{(5,6)}$. It causes cell damage by directly disrupting membrane function or indirectly damaging cell components. It has been shown in the literature that the measurement of lipid peroxidation level at first hours, 1 and 2 days after trauma, gives better results ${ }^{(7)}$. All these biochemical and cellular changes are observed in the first week of injury. At last, microglial cells and reactive astrocytes form a glial scar all over the lesion site. Regeneration of central axons along the lesion site is observed. Bare axons 
are seen after apoptosis of oligodendrocytes and wallerian degeneration. We should treat $\mathrm{SCl}$ effectively to improve the quality of life of patients. Therefore, we need to investigate appropriate therapeutic strategies to reduce the destruction of BSCB.

Green tea (Camellia sinensis) is a mixture of polyphenols, polysaccharides, thiamine, flavonoids, flavonols and amino acids, organic acids and vitamins ${ }^{(8)}$. It contains polyphenol and polyphenol oxidase enzymes. Catechins are a type of polyphenol and are the main antioxidant component in green tea. The most abundant catechins are epicatechin-3-gallate, epicatechin, epigallocatechin-3-gallate (EGCG) and epigallocatechin. Approximately $30-45 \%$ of the dry weight of green tea contains phenolic compounds. EGCG is the most common that constitutes approximately $50-80 \%$ of the total catechins $^{(9)}$. It has been shown in the literature that EGCG is protective against tumoral structures, especially with antimutagenic and antiproliferative action. The anti-inflammatory, antioxidant, antiallergic and neuroprotective effects of EGCG have been demonstrated in several in vitro and in vivo studies ${ }^{(10)}$.

EGCG, reportedly, has protective effects in maintaining bloodbrain barrier integrity by reducing caveolin-1 expression, downregulation of pro-inflammatory cytokines and by increasing the expression of proteins associated with a tight junction in the initial stage of brain ischaemia(11). These results showed that EGCGs could alter the permeability of BSCB after SCI. Thus, EGCGs can be considered as a potential neuroprotective agent against $\mathrm{SCl}$ because of its multiple protective effects on the neuronal injury, BSCB leakage, oedema and inflammation.

\section{MATERIALS AND METHODS}

First, the study protocol was approved by the ethics committee of our hospital, Ankara Training and Research Hospital, and the test procedures were performed in compliance with the study guides of the Animal Laboratory of the same hospital (decision no: 0309, date: 14.01.2009). Thirty-five male Wistar rats with an average weight of $210 \mathrm{~g}$ (200-220 g) were selected for the study. Adequate food and water were provided to the rats; they were then exposed to a 12-hour light cycle and 12-hour dark cycle in a standardised laboratory cage, and the ambient temperature was standardised at $18-21{ }^{\circ} \mathrm{C}, 40-60 \%$ humidity ${ }^{(12,13)}$.

Rats were divided into five groups: daily consumption group, treatment groups, saline group and control group. Each group consisted of seven rats. Other groups except the control group received compression-induced injury caused by clips closed with a pressure of $35 \mathrm{~g} / \mathrm{cm}^{2}$ for 1 minute. Group A, daily consumption group, was given $1.7 \mathrm{mg} / \mathrm{kg} /$ day EGCG dissolved in saline, intraperitoneally for 2 weeks to mimic daily the amount consumed. Group B was given $5 \mathrm{mg} / \mathrm{kg} /$ day EGCG (Sigma-Aldrich, Catalog No E4268 ${ }^{\circledR}$ ) for 7 days after $\mathrm{SCl}$. Group $\mathrm{C}$ received 10 mg/kg/day EGCG (Sigma-Aldrich, Catalog No E4268) for 1 week after $\mathrm{SCl}$. D group was the normal serum physiologic group; 0.25 cc normal saline was given intraperitoneally daily for 1 week after $\mathrm{SCl}$. SCI was not found in the control group, and no injection was administered ${ }^{(10)}$.

Samples were taken from all groups of rats after 28 days of trauma, and dry tissue samples were transferred to the biochemical analysis with $-4{ }^{\circ} \mathrm{C}$ cold chain. For histopathological examination, samples were determined by neutral formalin and were transferred to the hospital laboratory. Groups A, B and C were compared with saline-treated rats (group D) and control group (group E). 2'3'-cyclic nucleotide 3'-phosphodiesterase (CNP) (CNPase Ab-1), glial fibrillary protein (GFAP) (Cat. \#MS280-), nuclear factor (NF) (К) kappa (Cat. \#RB-1638) and endothelial nitric oxide synthase (eNOS) (Cat. \#RB-9279) primary antibodies were applied to the sections taken into the slides. Tissues were evaluated in the Leica DM 4000 image analysis system. Immunohistochemical markers such as CNP, eNOS, NF-K and GFAP were used to assess SCI injury and the efficacy of treatment.

\section{Statistical Analysis}

Data were expressed as mean \pm standard deviation. The statistical analysis was performed by using the t-test and X2test for SPSS Windows 13. Differences were noted significant if $p<0.05$.

\section{Surgical Procedure}

All rats were fasted overnight before the procedure. For anaesthesia, $10 \mathrm{mg} / \mathrm{kg}$ Ksilon (Rompun ${ }^{\circledR}, 2 \%$ solution, Bayer, İstanbul, Turkey) and $50 \mathrm{mg} / \mathrm{kg}$ ketamine hydrochloride (Ketalar ${ }^{\circledR}, 5 \%$ solution, Parke Davis-EWL, Eczacıbaşı, İstanbul, Turkey) intraperitoneal were applied ${ }^{(14)}$. The rats were placed in the prone position; the skin incision was then made along the dorsal midline, and the muscles were dissected and the vertebrae were clearly exposed. After that, a laminectomy was performed to expose the T9-T10 level without any damage to the dura mater and spinal cord. SCI was shown by compressing the spinal cord of each rat for 1 minute using an aneurysm clip (Tator clip) with a closing pressure of $35 \mathrm{~g} / \mathrm{cm}^{2(15)}$. The tissue was then closed anatomically. All rats were anaesthetised after 28 days, and samples of the damaged spinal cord, comprising the proximal and distal spinal cord sections of $0.5 \mathrm{~cm}$, were collected from previous incision sites. Finally, intra-atrial phenobarbital was applied, and the rats were sacrificed.

\section{Biochemical Examination}

The lipid peroxidation value per gram of tissue for each rat was calculated in nanomoles.

\section{Histology and Immunohistochemistry}

Tissue samples were divided into three parts, with each part consisting of $1 \mathrm{~mm}$. The fragments were fixed in $0.1 \mathrm{M}$ phosphate buffer $(\mathrm{pH}=7.4)$ containing $2.5 \%$ glutaraldehyde for 2 hours. The samples were then washed three times with buffer. After fixation, 1\% osmium tetroxide was used. Fixed tissues were dehydrated in alcohol. Lastly, the tissues were processed with propylene oxide. Then, it was mounted on tissue blocks using the Araldite CY212 kit. In the incubator, the hardened 
block from the polymerised tissues for 48 hours at $56^{\circ} \mathrm{C}$ was cut and made into semi-thin sections. It was then stained in the Toluidine Blue solution to examine by light microscopy. Thin sections obtained from these regions were stained with uranyl acetate and lead citrate and monitored by Carl Zeiss EVO LS 10 + ED transmission electron microscope and indicated by suitable magnifications.

Histopathological tissue samples were analysed under light microscopy. White-grey matter sparing, glial scar formation, protoplasmic astrocytes' number and cavity size were evaluated. Twelve or more microscopic domains were randomly selected from the spinal cord of each rat, and the degenerated axons were counted from the first right corner of the rectangular area to the last left corner in accordance with the protocol. Tissues were evaluated by two independent histopathologists who blinded this study.

\section{RESULTS}

Change in the body weight of rats was unnoticeable (approximately 1\%) throughout the study. Death associated with EGCG treatment was also not observed in the experiment.

\section{Biochemical Results}

Biochemically, the bioavailability of lipid peroxidation was measured. Statistical analysis of biochemical values showed no significant difference between group $D$ and groups $E, A$, $B$ and $C(p>0.05)$ (Table 1). However, a significant difference was found between groups $A, B$ and $C$ and group $D(p<0.05)$. Another study shows that daily and therapeutic doses of EGCG after $\mathrm{SCl}$ decrease the grade of lipid peroxidation statistically significantly (Figure 1).

\section{Histological Results}

In all rats, morphometric measurements were recorded to keep the preservation of the spinal cord simple after $\mathrm{SCl}$. In the EGCG-treated groups (A, B and C), the white matter of the spinal cord was essentially preserved in the cranial part of the spinal lesion, but it was not statistically significant. EGCG treatment provided more protection of grey matter in both the cranial and caudal parts of the spinal lesion than in the controls and was caudally significant at $5 \mathrm{~mm}$ from the centre of the lesion $(p<0.05)$. The volume of the cavities was alike in all three treated groups and not statistically significant. Glial scar formation was compared between EGCG-treated groups
(A, B and C) and saline-treated group (D). The glial scar site was larger in rats treated with saline $(D)$ than rats treated with EGCG (A, B and C) but not statistically significant $(p<0.05)$. To measure the axonal sprouting level, the number of GAP-43 positive fibres were examined. Axonal sprouting in EGCGtreated groups ( $A, B$ and $C$ ) was significantly higher than that of saline-treated rats $(p<0.05)$.

\section{Immunohistochemistry}

CNP immunostaining was used to assess oligodendrocyte distribution in the large and small magnified image in the medulla spinalis section. The control group (group E) was evaluated as normal $\mathrm{SCl}$-induced immunoreactivity. In groups, $B$ and C (EGCG-treated groups), oligodendrocytes around the tracts were observed to have regular localisation. In addition, CNP uptake in oligodendrocyte sections was more intense in both grey- and white matter than in the previous group. When these groups were compared with each other, no statistically significant difference was found. In group D, only traumainduced immunoreactivity was observed (Figure 2).

eNOS immunostaining of $\mathrm{D}$ group ( $\mathrm{SCl}+$ saline group) showed immunoreactivity in the cell membrane and cytoplasm of a few large neurons in medulla spinalis sections. In groups A, B and $\mathrm{C}$, eNOS reactivity was evident in the cell membrane and

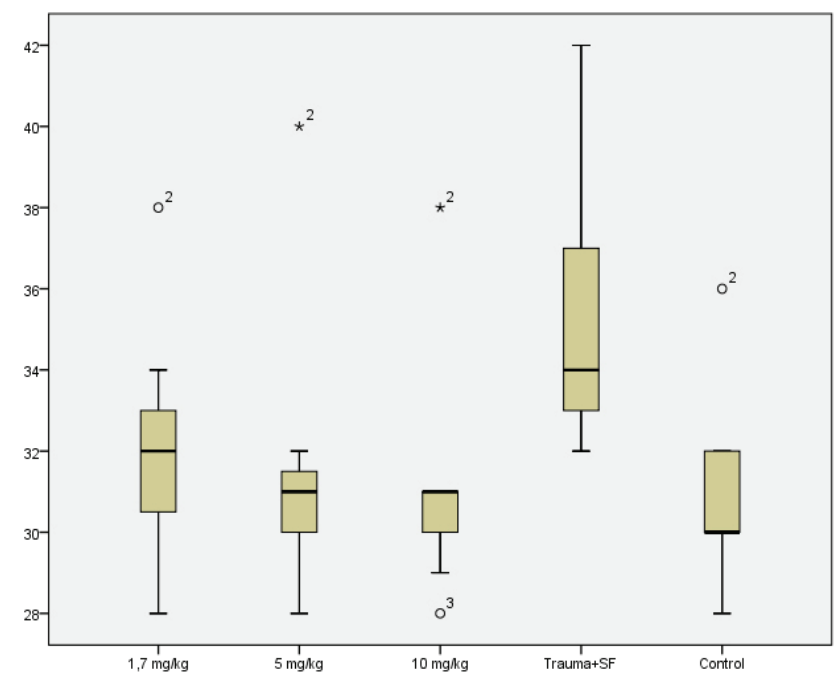

Figure 1. Multiple comparison of lipid peroxidation values between the $1.7 \mathrm{mg} / \mathrm{kg} /$ day, $5 \mathrm{mg} / \mathrm{kg}, 10 \mathrm{mg} / \mathrm{kg} /$ day trauma + saline, control groups respectively

Table 1. Distribution of biochemical values by groups

\begin{tabular}{lll}
\hline Groups & $\begin{array}{l}\text { Mean values of } \\
\text { raw data }\end{array}$ & $\begin{array}{l}\text { nmol/gr wet tissue/lipid } \\
\text { peroxidation mean values }\end{array}$ \\
\hline Group A & 0.165 & 32.443 \\
\hline Group B & 0.175 & 31.744 \\
\hline Group C & 0.164 & 31.258 \\
\hline Group D & 0.182 & 36.128 \\
\hline Group E & 0.153 & 30.063 \\
\hline
\end{tabular}


cytoplasm, similar to the D group. Cytoplasmic involvement was found to be relatively elevated in these four groups as compared to the control group. In group C, given high-dose EGCG, strong involvement of eNOS in neurons was observed at the membranous and cytoplasmic level.

GFAP immunostaining was used to monitor the distribution of astrocytes in the medulla spinalis sections. When the $D$ group was examined, it was determined that astrocytes with GFAP immunoreactivity were intense in the section. In the small magnified images, it was observed that the place of astrocytes in the vicinity of the capillary structures was interrupted. In the enlarged picture, the presence of very intense GFAP + astrocytes was observed around the large neurons. In groups A, B and C, GFAP + astrocytes, which shape the BSCB around the blood vessel, were interrupted occasionally. In group $C$, the central channel of the medulla spinalis section and the GFAP + astrocytes were observed in some areas in the grey matter (Figure 3).

$\mathrm{NF}$ immunostaining was performed to detect strong NF immunoreactivity in ependymal cells. In group D, NF immunoreactivity in ependymal cells was observed, and large neurons were found negative for NF. Some astrocytes showed NF involvement, and some astrocytes did not show immunoreactivity. It was noted in the A, B and C experimental groups that the involvement of the ependymal cells in the medulla spinalis sections increased significantly. Strong NF-K immunoreactivity in astrocytes was detected in large-scale examinations. Strong immunoreactivity was differentiated in

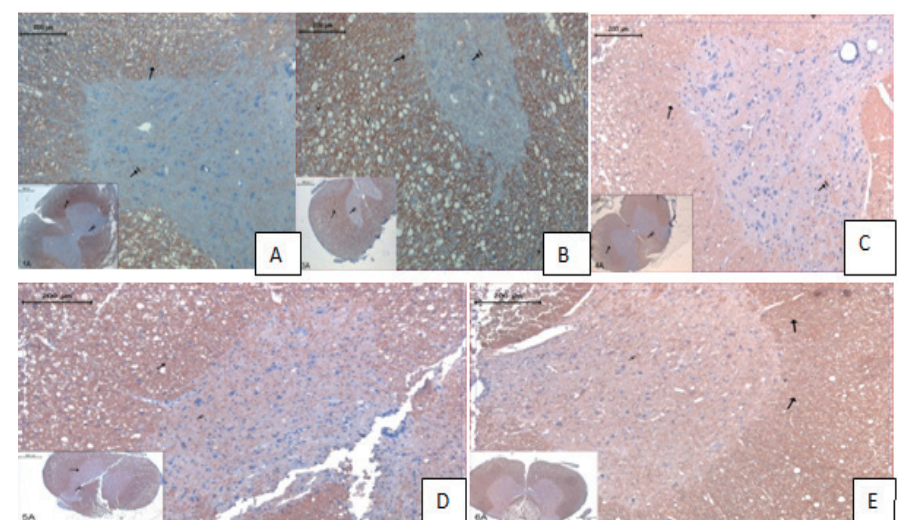

Figure 2. Oligodendrocytes is showed in medulla spinalis with CNP immunoreactivity; A) Control group, normal distrubition of parenchyma, B) In group D, normal configuration of medulla spinalis was impaired after injury, increased CNP immunopositivity was showed both white and grey matter, vacuolation was also prominent, C) In group A; Distribution of CNP reactivity is equally with normal tissue on the white and grey matter, a regular in habiting of oligodendrocytes was seen around of the tractus; D) : In group $B$; Immonoreactivity of oligodendrocytes with CNP was increased than group $A, E)$ In group C, CNP reactivity was similar with group $B$, but, it was showed that immunoreactivity was denser on the grey matter. $\rightarrow$ : CNP (+) axon in white matter and $\rightarrow$ I grey matter (Immunoperoxidase \& Hematoxylene A x 100 - B x 400)

CNP: 2'3'-cyclic nucleotide 3'-phosphodiesterase small neurons, whereas there was weak involvement in the perinuclear area in large neurons. On the other hand, cell membrane and peripheral cytoplasm were found to be weakly affected.

Clinical trials with CNP primary antibody demonstrated that myelinisation was raised at the grey matter in groups $A$ and $B$. Immunostaining with GFAP to evaluate the pattern of astrocytes showed a well perivascular organisation in group $B(5 \mathrm{mg})$ than group C (10 mg). These findings display that $5 \mathrm{mg}$ EGCG was associated with neuroprotective results, nevertheless, a dose of $10 \mathrm{mg}$ was associated with deterioration of the BSCB.

\section{DISCUSSION}

Traumatic $\mathrm{SCl}$ is a disease that causes serious mortality and morbidity in young people due to their aetiology ${ }^{(16)}$. There is still no common decision on treatment ${ }^{(17,18)}$. After $\mathrm{SCl}$, it causes autonomic dysfunction, motor paralysis and sensory anaesthesia under the lesion site. Like neuropathic pain, it can lead to a syndrome that greatly reduces the quality of life ${ }^{(19)}$. Experimental and clinical trials have showed the primary pathological changes that cause $\mathrm{SCl}$ symptoms, including neuropathic pain ${ }^{(4)}$. Post-SCl, oxidative stress-induced cell structure deterioration and ischaemia developed ${ }^{(19)}$. Antioxidants have the potential to prevent the tissue's harmful effect of the inflammatory reaction. In our study, EGCG, a polyhydroxy polyphenolic compound with antioxidant properties and present in the widely used green tea plant, was used in an $\mathrm{SCl}$ model with daily intake of $1.7 \mathrm{mg}$

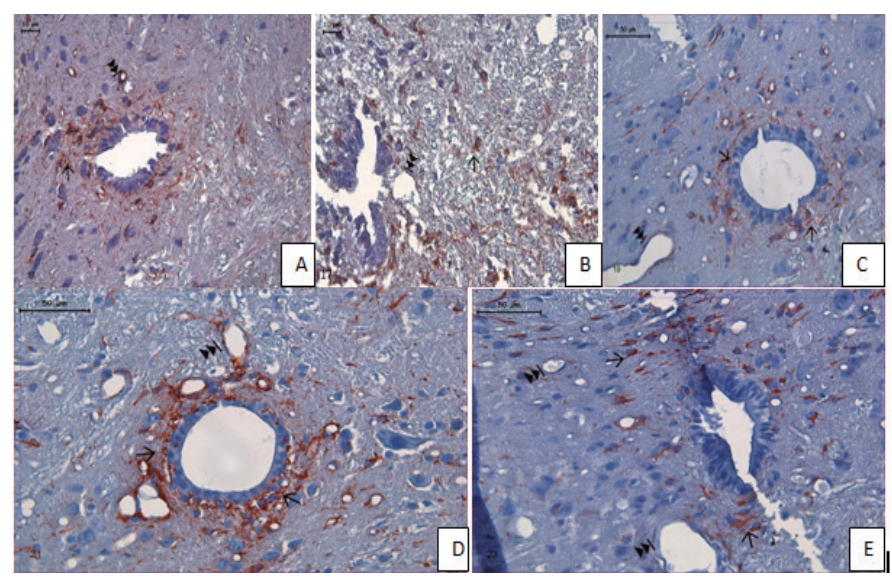

Figure 3. GFAP immunoreactivity was shown in the medulla spinalis sections, A) Control (group E), B) In group D, GFAP immunoreactivity of the astrocytes was widely. Arrangement of astrocytes was disrupted in patches around the capillary. GFAP (+) astrocytes was stronger around the large neurons, $C$ ) In group A, GFAP immunopostivity was denser than group $D$ and $E$, but blood-brain barrier was also disrupted, D) In group B showed similar findings with group A, contrary to expectations that blood-brain barrier was not healthy, E) In group C, GFAP reactivity was stronger than other groups, especially, around the central canal and grey matter, however, blood brain barrier is unhealed still; $\rightarrow$ : GFAP (+) Astrocytes, $M$ : Blood-brain barrier around the capillary (Immunoperoxidase \& Hematoxylene $x$ 400)

GFAP: Glial fibrillary protein 
$/ \mathrm{kg} /$ day and in treatment doses $(5 \mathrm{mg} / \mathrm{kg} /$ day and $10 \mathrm{mg} / \mathrm{kg} /$ day). EGCG has significant antioxidant activity on $\mathrm{SCl}$, measured by various biochemical assays. However, it has been shown to cause impairment in $\mathrm{BSCB}$, especially in the high-dose group $(10 \mathrm{mg} / \mathrm{kg})$.

Local tissue damage develops in the spinal cord after the primary mechanical impact. This damage causes interruption of ascending and descending nerve tracts in the spinal cord. Afterwards, a regular complex called secondary spinal cord injury ( $\mathrm{SSCl}$ ) leads to an increase in damage by initiating a cascade of biochemical, cellular and molecular mechanisms ${ }^{(18)}$. SSCl causes additional tissue loss and functional impairment ${ }^{(16)}$. Although these mechanisms were initiated during injury, they consisted of interacting, progressive chain reactions ${ }^{(17)}$. These reactions can lead to microvascular damage and cause endoneural oedema. Naturally, endoneural fluid pressure increases. Elimination of compression results in the resumption of oxygen and nutrient supplying blood flow. This rises the formation of lipid peroxidation and free oxygen radicals. The spinal cord is rich in lipids. In this case, lipid peroxidation is more severe than other tissues. Pro-inflammatory cytokines (e.g. IL-1, IL-6, TNF- $\alpha$ ), reactive oxygen species (ROS), proteases and glutamate excitotoxicity are responsible for the tissue damage, neuronal loss and axonal degeneration resulting in permanent neurological deficits ${ }^{(19)}$. Lipid peroxidation products disrupt cellular integrity by increasing membrane permeability for ions. These products also damage many functional components such as membrane proteins and enzymes ${ }^{(10)}$. Extracellular calcium enters the cell because of the damaged cell membrane and causes apoptosis and oedema.

EGCG increases nerve retention after $\mathrm{SCl}$ due to its antioxidant properties ${ }^{(20)}$. Biochemical analysis showed that myeloperoxidase activity in EGCG-treated rats was markedly lower than control group rats without EGCG. This showed that EGCG inhibited neutrophil infiltration in the injured spinal cord tissue and reduced the expression of pro-inflammatory agents $(\text { TNF- } \alpha, \text { IL1- } \beta \text {, iNOS and COX })^{(21)}$.

Experimental studies in rats showed that high-dose EGCG (50 $\mathrm{mg} / \mathrm{kg}$ ) resulted in severe liver necrosis and death. In addition, hepatotoxic effects were also observed in green tea extracts ${ }^{(22)}$. Conversely, there is proof that refined green tea extracts are hepatoprotective in certain doses in vivo. These study results analyse that the drug containing EGCG may be hepatotoxic or hepatoprotective depending on the administration and dose $^{(22)}$. In our study, the biochemical values of tissue samples were compared. The levels of lipid peroxidation in the treatment groups, namely, groups A, B and C, were shown to be statistically lower than the control group (group E) and the group treated with saline (group D). EGCG reduces secondary ischaemic damage following $\mathrm{SCl}$ as shown in the literature ${ }^{(21)}$. EGCG also reduces morbidity by reducing neurodegeneration associated with the spinal cord ischaemic process.

The histological and immunostaining studies showed that daily low-dose EGCG (group A) and low-dose (5 mg/kg/day) EGCG (group B) had less ROS formation and more neuroregeneration. In addition, it was shown to limit progressive damage and be neuroprotective due to SSCl. Immunostaining with GFAP was performed to evaluate the model of astrocytes. The evaluation showed that there was a better perivascular organisation in the group with low-dose $(5 \mathrm{mg} / \mathrm{kg}$ ) EGCG (group B) than highdose $(10 \mathrm{mg} / \mathrm{kg}$ ) EGCG (group C). These findings proved that the appropriate dose of EGCG was neuroprotective, but high-dose EGCG caused neurodegeneration and impairment of BSCB.

\section{CONCLUSION}

Although the exact cellular targets for polyphenol action are still uncertain, the mechanism of action seems to involve iron-chelating features and antioxidant-radical scavenging. This mechanism appears to be reasonable to clarify neuronal apoptosis experienced after $\mathrm{SCl}$. Our study indicated that steady injection of green tea (group A) and $5 \mathrm{mg} / \mathrm{kg}$ intake (group B) may augment regeneration after $\mathrm{SCl}$. Further studies are needed to confirm this polyphenol derivative to be recommended as a treatment of $\mathrm{SCl}$.

\section{Ethics}

Ethics Committee Approval: The study protocol was approved by the ethics committee of our hospital, Ankara Training and Research Hospital, and the test procedures were performed in compliance with the study guides of the Animal Laboratory of the same hospital (decision no: 0309, date: 14.01.2009).

Informed Consent: Since this study is an experimental study, informed consent was not obtained.

Peer-review: Internally peer-reviewed.

\section{Authorship Contributions}

Surgical and Medical Practices: A.D., Concept: R.A., Ö.M.U., A.D., G.T., Design: R.A., Ö.M.U., A.D., G.T., Data Collection or Processing:R.A.,Ö.M.U.,A.D.,G.T.,Analysis or Interpretation: R.A., Ö.M.U., A.D., G.T., Literature Search: R.A., Ö.M.U., A.D., G.T., Writing: R.A., Ö.M.U., A.D., G.T.

Conflict of Interest: No conflict of interest was declared by the authors.

Financial Disclosure: The authors declared that this study received no financial support.

\section{REFERENCES}

1. Tian ZR, Sharma A, Nozari A, Subramaniam R, Lundstedt T, Sharma HS. Nanowired drug delivery to enhance neuroprotection in spinal cord injury. CNS Neurol Disord Drug Target. 2012;11:86-95.

2. Menon PK, Muresanu DF, Sharma A, Mössler H, Sharma HS Cerebrolysin, a mixture of neurotrophic factors induces marked neuroprotection inspinal cord injury following intoxication of engineered nanoparticles from metals. CNS Neurol Disord Drug Target. 2012;11:40-9.

3. Bartanusz V, Jezova D, Alajajian B, Digicaylioglu M. The blood-spina cord barrier: morphology and clinical implications. Ann Neurol. 2011;70:194-206.

4. Silva NA, Sousa N, Reis RL, Salgado AJ. From basics to clinical: a comprehensive review on spinal cord injury. Prog Neurobiol. 2014; 114:25-57. 
5. Gutteridge JMC. Lipid peroxidation and antioxidants as biomarkers of tissue damage. Clin Chem. 1995;41:1819-28.

6. Cheeseman Kh, Slater TF. An introduction to free radical biochemistry. British Med Bulletin. 1993; 149:481-93.

7. Hall ED, Braughler M. Effects of intravenous methylprednisolone on spinal cord lipid peroxidation and Na-K ATPase activity. J Neurosurg. 1982;57:247-53.

8. Frei B, Higdon JV. Antioxidant activity of tea polyphenols in vivo: evidence from animal studies. J Nutr. 2003;133:3275S-84S.

9. Yilmaz Y. Novel uses of catechins in foods. Trends in Food Sci Technol. 2006; 17:64-71.

10. Sutherland BA, Rahman RMA, Appleton I. Mechanisms of action of gren tea catechins, with a focus on ischemiainduced neurodegeneration. J Nutr Biochem. 2006;17:291-306.

11. Zhang S, Liu Y, Zhao Z, Xue Y. Effects of green tea polyphenols on caveolin-1 of microvessel fragments in rats with cerebral ischemia. Neurol Res. 2010;32:963-70.

12. Macchi MM, Bruce JN. Human pineal physiology and functional significance of melatonin. Front Neuroendocrinol. 2004;25:177-95.

13. Persengiev S, Kanchev L, Vezenkova G. Circadian patterns of melatonin, corticosterone, and progesterone in male rats subjected to chronic stress: Effect of constant illumination. JPineal Res. 1991;11:57-62.

14. Zhang YL, Zhang PB, Qiu SD, Liu Y, Tian YF, Wang Y. Effects of ketamine-midazolam anesthesia on the expression of NMDA and AMPA receptor subunit in the peri-infarction of rat brain. Chin Med J. 2006;119:1555-62.
15. Rivlin AS, Tator $\mathrm{CH}$. Effect of duration of acute spinal cord compression in a new acute cord injury model in the rat. Surg Neurol. 1973; 10:38-43.

16. David S, Greenhalgh AD, López-Vales R. Role of phospholipase A2s and lipid mediators in secondary damage after spinal cord injury. Cell Tissue Res. 2012;349:249e267.

17. Hagg T, Oudega M. Degenerative and spontaneous regenerative processes after spinal cord injury. J Neurotrauma. 2006;23:264e280.

18. Cadotte DW, Fehlings MG. Spinal cord injury: a systematic review of current treatment options. Clin Orthop Rela T Res. 2011;469:732e741.

19. Khalatbary AR, Tiraihi T, Boroujeni MB, Ahmadvand H, Tavafi M, Tamjidipoor A. Effects of epigallocatechin gallate on tissue protection and functional recovery after contusive spinal cord injury in rats. Brain Res. 2010;1306:168-75.

20. Renno WM, Al-Khaledi G, Mousa A, Karam SM, Abul H, Asfar S. (-)-Epigallocatechin-3-gallate (EGCG) modulates neurological function when intravenously infused in acute and chronically injured spinal cord of adult rats. Neuropharmacology. 2014;77:100-19.

21. Khalatbary AR, Ahmadvand H. Anti-inflammatory effect of the epigallocatechin gallate following spinal cord trauma in rat. Iran Biomed J. 2011;15:31-7.

22. Xuczaj W, Skrzydlewska E. Antioxidative properties of black tea. Prev Med. 2005;40: 910-8. 